Document downloaded from:

http://hdl.handle.net/10251/60175

This paper must be cited as:

Lebtahi Ep-Kadi-Hahifi, L.; Patrício, P.; Thome, N. (2013). The special elements in a ring related to the Drazin inverses. Linear and Multilinear Algebra. 61(8):1017-1027. doi:10.1080/03081087.2012.728598.

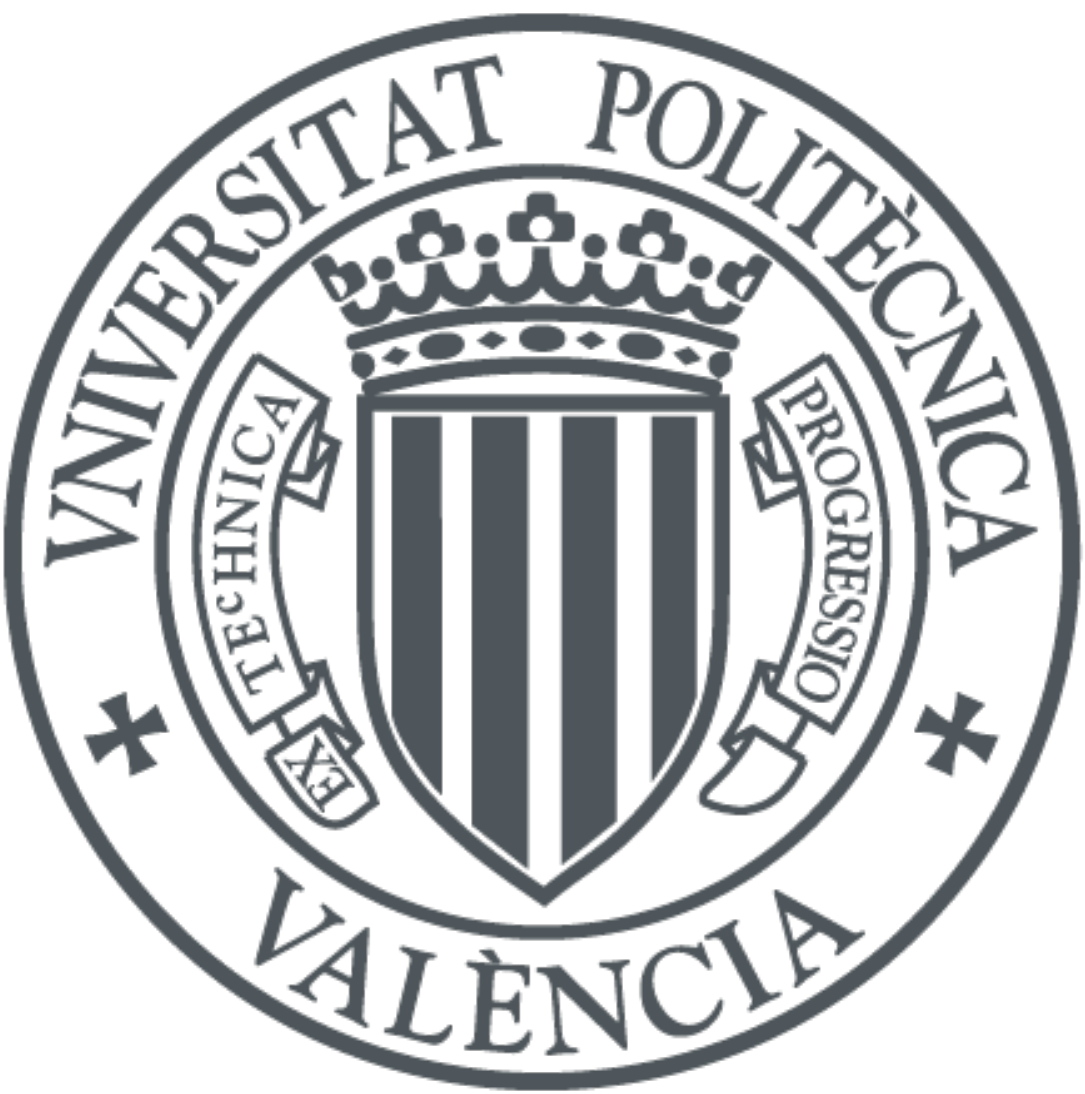

The final publication is available at

http://dx.doi.org/10.1080/03081087.2012.728598

Copyright Taylor \& Francis: STM, Behavioural Science and Public Health Titles

Additional Information 


\title{
The special elements in a ring related to Drazin inverses
}

\author{
Leila Lebtahi* $^{*} \quad$ Pedro Patrício $^{\dagger} \quad$ Néstor Thome* $^{*}$
}

\begin{abstract}
In this paper, the existence of the Drazin (group) inverse of an element $a$ in a ring is analyzed when $a^{m} k=k a^{n}$, for some unit $k$ and $m, n \in \mathbb{N}$. The same problem is studied for the case when $a^{*}=k a^{m} k^{-1}$ and for the $\{k, s+1\}$-potent elements. In addition, relationships with other special elements of the ring are also obtained.
\end{abstract}

Keywords: Involutory element; power; ring, Drazin inverse.

AMS subject classification: Primary: 15A09; Secondary: 16A30, 16E50

\section{Introduction}

Let $R$ be a ring with identity 1 . An element $k \in R$ is called involutory when $k^{2}=1$. We say $u \in R$ is regular if there exists an element $u^{-} \in R$ such that $u u^{-} u=u$. An involution $*$ on $R$ is an anti-automorphism of degree 2 , that is, $\left(a^{*}\right)^{*}=a,(a+b)^{*}=a^{*}+b^{*}$ and $(a b)^{*}=b^{*} a^{*}$ for all $a, b \in R$.

*Instituto Universitario de Matemática Multidiscilpinar. Universitat Politècnica de València. E-46022 Valencia, Spain. E-mail: \{leilebep,njthome\}@mat.upv.es. These authors have been partially supported by grant DGI MTM2010-18228, by Ministry of Education of Argentina (PPUA, grant Resol. 228, SPU, 14-15-222) and by Universidad Nacional de La Pampa, Facultad de Ingeniería (grant Resol. No 049/11).

†Departamento de Matemática e Aplicações, CMAT-Centro de Matemática, Universidade do Minho, 4710-057, Braga, Portugal. E-mail: pedro@math.uminho.pt. This author was financed by FEDER Funds through "Programa Operacional Factores de Competitividade - COMPETE" and by Portuguese Funds through FCT - "Fundação para a Ciência e a Tecnologia", within the project PEst-C/MAT/UI0013/2011. 
An element $a \in R$ is called hermitian if $a^{*}=a$, unitary if $a a^{*}=a^{*} a=1$ and normal if $a a^{*}=a^{*} a$.

We will make use of the right and left ideals generated by $a$, defined as $a R=\{a x: x \in R\}$ and $R a=\{x a: x \in R\}$ respectively.

The Drazin inverse of $a$, when it exists, is denoted by $a^{D}$, and it is defined as the unique solution to $a^{D} a a^{D}=a^{D}, a a^{D}=a^{D} a, a^{r+1} a^{D}=a^{r}$ for some nonnegative integer $r$. The smallest nonnegative integer $r$ that fulfills $a^{r} \in$ $a^{r+1} R \cap R a^{r+1}$ is called the Drazin index of $a$ and is denoted by $\operatorname{ind}(a)$. When $\operatorname{ind}(a) \leq 1$, we say $a$ has a group inverse, denoted by $a^{\#}$. That is, $a^{\#}$ satisfies $a a^{\#} a=a, a^{\#} a a^{\#}=a^{\#}, a a^{\#}=a^{\#} a$.

An element $a \in R$ is Moore-Penrose invertible if it exists $a^{\dagger} \in R$ such that $a a^{\dagger} a=a, a^{\dagger} a a^{\dagger}=a^{\dagger},\left(a a^{\dagger}\right)^{*}=\left(a a^{\dagger}\right)$ and $\left(a^{\dagger} a\right)^{*}=a^{\dagger} a$.

We say a ring $R$ is Dedekind finite if $u v=1$ implies $v u=1$. A characterization of a Dedekind finite ring $R$ is the following: given two regular $u, v \in R$, the conditions $u \sim v$ and $u R \subseteq v R$ imply $u R=v R$, where $u \sim v$ means $u R \cong v R$ as right $R$-modules.

Let $k \in R$ be a unit and $s \in \mathbb{N}$. An element $a \in R$ is called $\{k, s+1\}$ potent if it satisfies

$$
k a^{s+1} k^{-1}=a .
$$

Besides this special element, we will study the elements $a \in R$ satisfying

$$
a^{m} k=k a^{n}, \quad m, n \in \mathbb{N} \text { for some unit } k \in R
$$

and the elements $a \in R$ satisfying

$$
a^{*}=k a^{m} k^{-1}, \quad m \in \mathbb{N}, m>1 \text { for some unit } k \in R .
$$

Furthermore, we will consider a ring version of $k$-EP [7]: given $k \in R$ we say $a$ is $k-E P$ if

$$
a R=k a^{*} R .
$$

For related topics we refer the reader to $[1,4,5,6,8]$.

This paper is organized as follows. In Section 2, some basic results about $\{k, s+1\}$-potent elements are presented. In Section 3, the index and the existence of the Drazin inverse of an element $a$ satisfying (2) is analyzed. In addition, the same problem is studied for an element such that (3) holds. Some representations for the Drazin inverses have been obtained. Relationships with another special elements are found in Section 4. Finally, in Section 5 , further remarks and open problems are presented. 


\section{Basic properties}

In the complex matrices approach, some results for $\{k, s+1\}$-potent matrices have been given in [5].

The first question in the ring setting is related to the existence of the $\{k, s+1\}$-potent elements. It is easy to see that for each unit element $k$ and for each $s \in \mathbb{N}$, we have $\left\{a \in R: k a^{s+1} k^{-1}=a\right\} \neq \emptyset$.

A important particular case is when $k$ is involutory. How to use the element $k$ when $k$ is involutory?

Lemma 1 Let $k \in R$ be a unit, $s \in \mathbb{N}$ and $a \in R$. Then the following conditions are equivalent:

(a) a is $\{k, s+1\}$-potent.

(b) $k^{-1} a k=a^{s+1}$.

(c) $k^{-1} a=a^{s+1} k^{-1}$.

(d) $a k=k a^{s+1}$.

Proof. The equivalences can be easily obtained from the definition.

In addition, we present basic properties showing situations when the set of $\{k, s+1\}$-potent elements is closed under certain operations.

Lemma 2 Let $k \in R$ be a unit, $s \in \mathbb{N}$ and $a, b \in R$ be two $\{k, s+1\}$-potent elements. The following properties hold.

(a) If $s=1$ then $a b=-b a$ if and only if $a+b$ is $\{k, 2\}$-potent.

(b) If $a b=b a=0$ then $a+b$ is $\{k, s+1\}$-potent.

(c) If $a b=b a$ then $a b$ is $\{k, s+1\}$-potent.

(d) If $a$ is a unit then $a^{-1}$ is $\{k, s+1\}$-potent.

(e) If $w \in R$ is a unit and $k w=w k$ then $w a w^{-1}$ is $\{k, s+1\}$-potent.

(f) If $k$ is hermitian then $a^{*}$ is $\left\{k^{-1}, s+1\right\}$-potent. 
Proof. All these properties can be easily obtained by definition.

By using unitary similarity the next results allow us to construct some more examples from an initial one.

Lemma 3 Let $k \in R$ be an involutory hermitian element, $s \in \mathbb{N}$ and $a \in R$ be $\{k, s+1\}$-potent. If $u \in R$ is unitary such that $k u=u k$ then uau ${ }^{*}$ is $\{k, s+1\}$-potent.

Proof. Since $k a^{s+1} k^{-1}=a$ and $k^{-1}=k=k^{*}$ we get

$$
k\left(u a u^{*}\right)^{s+1} k^{-1}=k u a^{s+1} u^{*} k^{*}=u k a^{s+1} k^{*} u^{*}=u a u^{*},
$$

and then the result has been shown.

We will now focus on the following question: Does any $\{k, s+1\}$-potent element has this form? That is, for two given $\{k, s+1\}$-potent elements $a$ and $b$, is it possible to find a unitary $u$ such that $b=u a u^{*}$ and $k u=u k$ ? The answer is negative as the following counterexample shows: in the ring of $2 \times 2$ complex matrices,

$$
A=\left[\begin{array}{rr}
0 & 0 \\
0 & -i
\end{array}\right] \quad \text { and } \quad B=\left[\begin{array}{ll}
1 & 0 \\
0 & i
\end{array}\right]
$$

are $\{K, 5\}$-potent for $K=I_{2}$, but it is easy to check that there is no unitary matrix $U$ such that $B=U A U^{*}$. This example also solves the same problem for nonsingular matrices (see Lemma 2 (e)).

Lemma 4 If $a \in R$ is $\{k, s+1\}$-potent then the following equalities hold

$$
a^{(s+1)^{2}}=k^{-1} a^{s+1} k=k^{-2} a k^{2} .
$$

Proof. It is clear that the definition yields to

$$
a^{(s+1)^{2}}=\left(a^{s+1}\right)^{s+1}=\left(k^{-1} a k\right)^{s+1}=k^{-1} a^{s+1} k .
$$

The second equality follows from (5) and the definition.

Lemma 5 Let $k \in R$ be involutory, $s \in \mathbb{N}$ and $a \in R$ be $\{k, s+1\}$-potent. Then the following conditions hold: 
(a) $k a^{s+2}=a^{s+2} k$.

(b) $a^{s+2}=(k a)^{2}=(a k)^{2}$.

(c) $(k a)^{2 s+1}=k a$ and $(a k)^{2 s+1}=a k$.

Proof. We have that

$$
k a^{s+2}=k a^{s+1} a=a k a=a a^{s+1} k=a^{s+2} k
$$

which yields (a). By using this property we get

$$
a^{s+2}=k a^{s+2} k=k a a^{s+1} k=k a(k a)=(k a)^{2}
$$

which gives (b). In order to show (c) we use (b) to obtain

$$
(k a)^{2 s+1}=k a\left((k a)^{2}\right)^{s}=k a\left(a^{s+2}\right)^{s}=k a^{(s+1)^{2}}=k a .
$$

\section{Group and Drazin inverses}

As a direct consequence of the definition of the group inverse we have the following result which will be useful.

Lemma 6 Let $a \in R$ and $l \in\{1,2,3, \ldots\}$. Then $a^{l+2}=a$ if and only if there exists $a^{\#}$ and $a^{\#}=a^{l}$.

What about the index of $a$ when $a^{m} k=k a^{n}$ where $k$ is a unit?

Theorem 1 Let $R$ be a Dedekind finite regular ring. If $a \in R$ is such that $a^{m} k=k a^{n}$ for some unit $k$ with $m, n \in \mathbb{N}, m \neq n$, then $\operatorname{ind}(a) \leq \min \{m, n\}$.

Proof. Assume $n>m$. Applying the definition we get $a^{n} R \subseteq a^{m} R=$ $a^{m} k R=k a^{n} R$. It is easy to see that the function $\phi: k a^{n} R \rightarrow a^{n} R$ defined by $\phi(x)=k^{-1} x$ is a (right) ideal isomorphism, that is $k a^{n} R \simeq a^{n} R$. Using the finiteness of $R$, this ensures that $a^{m} R=a^{n} R$. Hence, by [2, Theorem 2] $\operatorname{ind}(a) \leq m$. The case $m>n$ is similar by using $k^{-1} a^{m}=a^{n} k^{-1}$. 
When $m=n$, if $C(k)=\{b \in R: b k=k b\}$ is the centralizer of $k$, we have that the problem $a^{m} k=k a^{m}$ is reduced to $a^{m} \in C(k)$.

In the previous theorem we may assume that appropriate powers of $a$ are regular instead of the general regularity condition. Nevertheless, we remark that an element may be regular without having a regular power as showed in $[3]$.

In order to show the existence of the group inverse of an element $a \in R$ we give the following result.

Corollary 1 Let $R$ be a Dedekind finite ring. If $a \in R$ is regular and $\{k, s+$ $1\}$-potent then there exists the group inverse of $a$.

Proof. The existence of the group inverse of $a$ is guaranteed by Theorem 1 because $\operatorname{ind}(a) \leq 1$.

Lemma 7 Let $a \in R$ be $a\{k, m\}$-potent element with $m>1$. Let $l \in \mathbb{N}$ such that $m$ divides $l+2$ and $m \neq l+2$. Then the following conditions are equivalent:

(a) $a^{\#}=a^{l}$.

(b) $k^{2} a^{m}=a^{\frac{l+2}{m}} k^{2}$.

(c) $k a=a^{\frac{l+2}{m}} k$.

Proof. We are going to show $(a) \Longleftrightarrow(b)$. Suppose that (a) holds. By Lemma $6, a^{l+2}=a$. Thus, by definition we get

$$
k a^{m} k^{-1}=a=a^{l+2}=k^{-1}\left(k a^{l+2} k^{-1}\right) k=k^{-1}\left(k a^{m} k^{-1}\right)^{\frac{l+2}{m}} k=k^{-1} a^{\frac{l+2}{m}} k
$$

Hence, $k^{2} a^{m}=a^{\frac{l+2}{m}} k^{2}$. Then, (b) is obtained.

Conversely, from $k^{2} a^{m}=a^{\frac{l+2}{m}} k^{2}$ and $k a^{m} k^{-1}=a$ we get $a=k a^{m} k^{-1}=$ $k^{-1} a^{\frac{l+2}{m}} k$. Then

$$
a^{l+2}=k\left(k^{-1} a^{\frac{l+2}{m}} k\right)^{m} k^{-1}=k a^{m} k^{-1}=a
$$

Then, by Lemma 6 we get $a^{l}=a^{\#}$. The equivalence between (b) and (c) is evident. 
Corollary 2 If $a \in R$ is $\{k, s+1\}$-potent then the following conditions are equivalent:

(a) There exists $a^{\#}$ and $a^{\#}=a^{(s+1)^{2}-2}$.

(b) $k^{2} a^{s+1}=a^{s+1} k^{2}$.

(c) $k a=a^{s+1} k$.

(d) $k^{2} a=a k^{2}$.

Proof. (b) $\Rightarrow$ (d) Since $k^{2} a^{s+1} k^{-2}=a^{s+1}$, we get

$$
k^{-2} a k^{2}=a^{(s+1)^{2}}=\left(k^{2} a^{s+1} k^{-2}\right)^{s+1}=k^{2} a^{(s+1)^{2}} k^{-2}=a
$$

by Lemma 4 .

(d) $\Rightarrow$ (b) Since $k a k^{-1}=k^{-1} a k$, by (1) we get

$$
k^{2} a^{s+1} k^{-2}=k a k^{-1}=k^{-1} a k=a^{s+1} .
$$

In order to show the equivalences (a) $\Leftrightarrow$ (b) $\Leftrightarrow$ (c) it is sufficient to apply Lemma 7 with $m=s+1$ and $l=(s+1)^{2}-2$.

Lemma 8 If $a \in R$ is $\{k, s+1\}$-potent with $k$ involutory then the following (equivalent) conditions hold

(a) $a^{(s+1)^{2}}=a$.

(b) $a^{\#}=a^{(s+1)^{2}-2}$.

In this case, $a^{\#}$ is $\{k, s+1\}$-potent.

Proof. In order to obtain item (a), it is sufficient to substitute $k^{2}=1$ in Lemma 4. The property $a^{\#}=a^{(s+1)^{2}-2}$ is derived from (a) and Lemma 6 . Moreover, by item (c) of Lemma 2, the fact that $a^{\#}$ is $\{k, s+1\}$-potent can be obtained. 
Remark 1 We observe that none of the equivalent conditions given in the Lemma 8 imply that $a$ is $\{k, s+1\}$-potent. In fact, in the ring of $2 \times 2$ complex matrices, if $s=1$ and

$$
A=\left[\begin{array}{ll}
1 & 0 \\
0 & \alpha
\end{array}\right]
$$

with $\alpha \in \mathbb{C}-\{1\}$ such that $\alpha^{3}=1$, it is clear that $A^{4}=A$ and $K A^{2} K \neq A$ for any involutory matrix $K \in \mathbb{C}^{2 \times 2}$. The same example can be used to assure that neither in Lemma 7 nor in Corollary 2 the converse is true.

Theorem 2 Let $k \in R$ be involutory, $s \in \mathbb{N}$ and $a \in R$ be $\{k, s+1\}$-potent. Then

$$
a^{\#}=(k a)^{2 s-1} k \text {. }
$$

Proof. By Lemma 5 and Lemma 6 , we have that $(k a)^{\#}=(k a)^{2 s-1}$. Now, by using $s$ times that $a$ is $\{k, s+1\}$-potent we get

$$
(k a)^{2 s-1}=\left((k a)^{2}\right)^{s-1} k a=\left(a^{s+2}\right)^{s-1} k a=\left(a^{s+2}\right)^{s-1} a^{s+1} k=a^{(s+1)^{2}-2} k=a^{\#} k
$$

by Lemma 8 . Finally, $a^{\#}=(k a)^{2 s-1} k$.

Lemma 9 If $a, k \in R$ such that $k$ is a unit and $k^{2} a=a k^{2}$. Then the following conditions hold:

(a) $a$ is $\{k, s+1\}$-potent if and only if $k^{-1} a^{s+1} k=a$.

(b) $k^{2} a^{s+1}=a^{s+1} k^{2}$.

(c) if a is $\{k, s+1\}$-potent then $k^{2} a^{\#}=a^{\#} k^{2}$.

Proof. Since $k$ is a unit, the assumption $k^{2} a=a k^{2}$ implies that $k^{-1} a k=$ $k a k^{-1}$. Then $k^{-1} a^{s+1} k=k a^{s+1} k^{-1}$, from which (a) and (b) can be deduced. The third condition follows directly from Corollary 2.

Theorem 3 Let $R$ be a ring with $k \in R$ such that $k^{t}=1$ for some $t \in \mathbb{N}$, $t>1$. If $a \in R$ satisfies that $a^{m} k=k a^{n}$ with $m, n \in \mathbb{N}$. Then

(a) $a^{m^{t}}=a^{n^{t}}$. 
(b) There exists $a^{D}$ and

$$
a^{D}=\left\{\begin{array}{lll}
a^{m^{t}-n^{t}-1} & \text { if } & m>n \sqrt[t]{3} \\
a^{n^{t}-m^{t}-1} & \text { if } & n>m \sqrt[t]{3}
\end{array} .\right.
$$

Proof. (a) By definition

$a^{m^{2}}=\left(k a^{n} k^{-1}\right)^{m}=\left(k a^{m} k^{-1}\right)^{n}=\left(k^{2} k^{-1} a^{m} k k^{-2}\right)^{n}=\left(k^{2} a^{n} k^{-2}\right)^{n}=k^{2} a^{n^{2}} k^{-2}$

Following a similar reasoning up to the power $t$ we obtain item (a) because $k^{t}=1$.

(b) Assume that $m>n \sqrt[t]{3}$. The existence of the Drazin inverse of $a$ follows from

$$
a^{n^{t}} \in a^{n^{t}} R \cap R a^{n^{t}}=a^{m^{t}} R \cap R a^{m^{t}} \subseteq a^{n^{t}+1} R \cap R a^{n^{t}+1}
$$

since $m>n$. In order to compute the Drazin inverse of $a$ it is necessary to find $\left(a^{n^{t}}\right) \#$. We are going to check that $\left(a^{n^{t}}\right)^{\#}=a^{m^{t}-2 n^{t}}$. In fact,

$$
\left(a^{n^{t}}\right)^{2} a^{m^{t}-2 n^{t}}=a^{m^{t}}=a^{n^{t}}
$$

and

$$
a^{m^{t}-2 n^{t}} a^{n^{t}} a^{m^{t}-2 n^{t}}=a^{m^{t}} a^{m^{t}-3 n^{t}}=a^{n^{t}} a^{m^{t}-3 n^{t}}=a^{m^{t}-2 n^{t}} .
$$

Then,

$$
a^{D}=a^{n^{t}-1}\left(a^{n^{t}}\right) \#=a^{m^{t}-n^{t}-1} .
$$

The remaining case is obtained in a similar way.

We recall that $a \in R$ is $E P$ if $a a^{\dagger}=a^{\dagger} a$. This is equivalent to $a^{\dagger}$ exists and $a R=a^{*} R$.

Lemma 10 If $a \in R$ is a Moore-Penrose invertible then $a^{*} R \cong a R$ as right $R$-modules.

Proof. The isomorphism is given by $\Psi: a^{*} R \rightarrow a R$ defined as $\Psi(x)=\left(a^{\dagger}\right)^{*} x$.

Proposition 1 Let $R$ be a Dedekind finite ring and $a \in R$ be a MoorePenrose invertible element which is $k$-EP. The following hold. 
(a) If $k a^{*}$ is regular then $\left(k a^{*}\right)^{\#}$ exists and $\left(k a^{*}\right)^{\#}=k a^{*}\left(w a^{\dagger}\right)^{2}$ for some $w$ such that $a=k a^{*} w$.

(b) If $k$ is a unit then $k a^{*} R=\left(k a^{*}\right)^{*} R$.

(c) If $k a^{*}$ is Moore-Penrose invertible then $k a^{*}$ is EP.

Proof. (a) Since $a^{\dagger}$ exists, we get $a \in R a^{*} a$ from which $a^{*} \in a^{*} a R=a^{*} k a^{*} R$. Therefore, $k a^{*} \in k a^{*} k a^{*} R=\left(k a^{*}\right)^{2} R$. As $R$ is Dedekind finite, this implies $\left(k a^{*}\right)^{\#}$ exists by Theorem 2 (xi) in [2]. Assume $w$ is such that $a=k a^{*} w$. Since $a^{*}=a^{*} a a^{\dagger}=a^{*} k a^{*} a w a^{\dagger}$, we get the equality $k a^{*}=\left(k a^{*}\right)^{2} w a^{\dagger}$. From this and [3, Lemma 3.3] we obtain the expression $\left(k a^{*}\right)^{\#}=k a^{*}\left(w a^{\dagger}\right)^{2}$.

(b) Since $k$ is a unit and $a$ is $k-E P$, the equalities $k^{*} a R=a R=a k^{*} R=$ $\left(k a^{*}\right)^{*} R$ hold.

(c) Suppose $k a^{*}$ is Moore-Penrose invertible. Lemma 10 implies that

$$
k a^{*} R=a R \supseteq a k^{*} R=\left(k a^{*}\right)^{*} R \cong k a^{*} R
$$

hold. From the Dedekind finiteness of the ring we obtain $\left(k a^{*}\right)^{*} R=k a^{*} R$. As $\left(k a^{*}\right)^{\dagger}$ exists, the result follows.

Another problem related to the previous ones is the following: $a^{*}=$ $k a^{m} k^{-1}$ where $k$ is a unit and $m>1$. The case $m=1$ will be considered in the next section.

Theorem 4 Let $R$ be a Dedekind finite ring. If $a \in R$ is regular and satisfies $a^{*}=k a^{m} k^{-1}$ with $k$ a unit and $m>1$ then there exists $a^{\#}$. Moreover, if $a^{\dagger}$ exists then $a^{*}$ is $k$-EP, $k a$ is group invertible with $(k a)^{\#}=k a\left(a^{m-1} k^{-1}\left(a^{*}\right)^{\dagger}\right)^{2}$ and $a^{\#}=a^{m^{2}-2}$ when $k$ is hermitian or involutory.

Proof. Since $a=\left(k^{-1}\right)^{*}\left(a^{m}\right)^{*} k^{*}$, we get $a^{*}=k\left(\left(k^{-1}\right)^{*}\left(a^{m}\right)^{*} k^{*}\right)^{m} k^{-1}=$ $k\left(k^{-1}\right)^{*}\left(a^{m^{2}}\right)^{*} k^{*} k^{-1}$. Then

$$
a=\left(k^{-1} k^{*}\right)^{-1} a^{m^{2}} k^{-1} k^{*} .
$$

Denoting $t=\left(k^{-1} k^{*}\right)^{-1}$ we get that $a$ is $\left\{t, m^{2}\right\}$-potent. Corollary 1 assures the existence of $a^{\#}$ and Lemma 8 gives the desired expression.

Finally, from $a^{*}=k a^{m} k^{-1}$ and the existence of $a^{\#}$ we obtain $a^{*} R=$ $k a^{m} R=k a R$, that is, $a^{*}$ is $k-E P$. Furthermore, the invertibility of $k$ together with the existence of $a^{\dagger}$ make $a k$ regular. The group invertibility of $k a$ follows from Proposition 1. In addition, writing $a^{*}=k a w$ with $w=a^{m-1} k^{-1}$, the expression for $(k a)^{\#}$ follows by Proposition 1. 


\section{Relationships with other elements in $R$}

Let $k \in R$ be an invertible element and $a \in R$. Let us consider the following conditions:
(A) $k a^{s+1} k^{-1}=a$.
(B) $a^{s+3}=a$.
(C) $a^{\#}=a^{s+1}$.
(D) $a^{s+1}=a^{*}$.
(E) $k a^{*} k^{-1}=a$.
(F) $a a^{*}=a^{*} a=1$.
(G) $a=k b$ for some $b \in R$ such that $b^{2}=1$.

The following theorems can be proved by using similar techniques to those used in the previous results.

Theorem 5 The following statements hold:

(i) $(A)+(E) \Longrightarrow(D)$.

(ii) $(A)+(D) \Longrightarrow(E)$.

(iii) $(D)+(E) \Longrightarrow(A)$.

(iv) $(A)+(D)+(E)=(A)+(E)=(A)+(D)=(D)+(E)$.

(v) $(B)+(F) \Longrightarrow(D)$.

Theorem 6 Let $k \in R$ be an involutory element. The following statements hold:

(i) $(A)+(E)+a$ unit $\Longrightarrow a^{s}$ satisfies $(F)$.

(ii) $(F)+(G) \Longrightarrow(E)$.

(iii) $(E)+(F) \Longrightarrow(G)$.

(iv) $(E)+(G) \Longrightarrow(F)$. 
(v) $(E)+(F)+(G)=(E)+(F)=(E)+(G)=(F)+(G)$.

(vi) $(A)+(G) \Longrightarrow a^{s+2}=1$.

(vii) $(B)+(G) \Longrightarrow(A)$.

Theorem 7 Let $a \in R$ satisfying $(F)$. Then following conditions are equivalent:

(i) a is $\{k, s+1\}$-potent.

(ii) there exists $b \in R$ such that $b$ is $\{k, s+1\}$-potent and $b^{*}=a$.

Theorem 8 Let $a, k \in R$ such that $k$ is involutory. Then following conditions are equivalent:

(i) a is $\left\{k^{*}, s+1\right\}$-potent.

(ii) there exists $b \in R$ such that $b$ is $\{k, s+1\}$-potent and $b^{*}=a$.

Theorem 9 Let $a, k \in R$ such that $k$ is hermitian involutory and a is $\{k, s+$ $1\}$ potent. Then following conditions are equivalent:

(i) a satisfy (D).

(ii) there exists $h \in R$ hermitian such that $a=k h$.

Proof. We assume that $k^{2}=1, k^{*}=k$ and $k a^{s+1} k=a$. Then

$$
a^{s+1}=a^{*} \Longleftrightarrow k a k=a^{*} \Longleftrightarrow a=k\left(a^{*} k\right) \Longleftrightarrow a=k h \text { with } h^{*}=h
$$

\section{$5 \quad$ Final remarks and questions}

In this paper, we consider results related to group and Drazin inverses of elements in a ring with identity. Most of the results presented are already known for complex matrices and some of them for bounded operators on Hilbert spaces. However, we use a different technique in order to extend the results. In complex matrix theory the main tool for providing the existence 
of the group or Drazin inverse is the rank. In the case of bounded operators of Hilbert spaces, the method of operator matrices is a powerful tool. In this paper, we apply a purely algebraic technique.

The ring $R$ is $*$-reducing if the $*$-cancelation property holds for all $a \in R$, that is, $c a a^{*}=b a a^{*}$ implies $c a=b a$ for all $b, c \in R$. In a *-reducing ring $R$, it can be proved that for an idempotent element $e \in R$, the conditions $e$ normal and $e$ hermitian are equivalent (because if $e e^{*}=e^{*} e$ then $e^{\#}=e^{\dagger}=e$ and so $\left.e^{*}=e^{\dagger} e^{*} e=e e^{*} e=e^{2} e^{*}=e e^{*}\right)$. In addition, a more general result can be stated in the matrix context but not in the wider context of rings as we can see in the item (IV) below.

We note that given a unitary $k, a$ is $k^{*}-E P$ iff $a^{*}$ is $k-E P$.

In the approach of complex square matrices, the following results have been proved in [5]:

(I) If $A$ is a normal $\{K, s+1\}$-potent matrix with $K^{2}=I$, then $A^{*}=$ $A^{(s+1)^{2}-2}$. However, in rings theory (even commutative rings!) that result does not work. As a counterexample we consider the commutative ring

$$
R=\left\{\left[\begin{array}{ll}
x & y \\
0 & x
\end{array}\right]: x, y \in \mathbb{C}\right\}
$$

equipped with the involution $*: R \rightarrow R$ given by

$$
\left[\begin{array}{ll}
x & y \\
0 & x
\end{array}\right]^{*}=\left[\begin{array}{rr}
x & -y \\
0 & x
\end{array}\right] .
$$

In this case, considering the matrix $A=a I_{2}, s \geq 3$ the smallest nonnegative integer satisfying $a^{s}=1$ and $K$ any nonsingular matrix we get that $A^{*}=A \neq A^{s-1}=A^{(s+1)^{2}-2}$.

(II) If $A$ is a $\{K, s+1\}$-potent matrix with $K^{2}=I$ and there is a hermitian matrix $H$ such that $A=K H$ then there exists a tripotent matrix $B$ satisfying $A=K B$. Again, in ring theory (even commutative rings!) that result does not hold. As a counterexample we consider over the commutative ring $\mathbb{Z}_{14}$ with the identity as the involution: $a=2, k=1$, $h=2$. In this case, $k a^{4} k^{-1}=a, a=k h$ with $h^{*}=h$ and however if $a=k b$ for some tripotent $b \in \mathbb{Z}_{14}$ then $2=b=2^{3}=8$, which is a contradiction.

(III) If $A$ is a $\{K, s+1\}$-potent matrix with $K^{2}=I$ and there is a normal matrix $N$ such that $A=K N$ then there exits a matrix $T$ such that 
$A=K T$ with $T^{*}=T^{2 s-1}$. Once again, this result does not work in a (commutative) ring. For that, if, in the ring $\mathbb{Z}_{14}$, we consider $a=2$, $k=1, s=3$ and $b=2$ then the existence of $t$ such that $a=k t$ with $t^{*}=t^{5}$ yields a contradiction.

(IV) If $A$ is a $\{K, 2\}$-potent matrix with $K^{2}=I$ then there is a normal matrix $N$ such that $A=K N$ if and only if there is a hermitian matrix $H$ such that $A=K H$. In ring theory, this result does not work. As a counterexample we consider the ring

$$
R=\left\{\left[\begin{array}{ll}
x & y \\
0 & z
\end{array}\right]: x, y, z \in \mathbb{Z}_{6}\right\}
$$

equipped with the involution $*: R \rightarrow R$ given by

$$
\left[\begin{array}{ll}
x & y \\
0 & z
\end{array}\right]^{*}=\left[\begin{array}{ll}
z & y \\
0 & x
\end{array}\right]
$$

In this case, considering the matrix $A=\left[\begin{array}{ll}3 & 0 \\ 0 & 1\end{array}\right]$ and $K=\left[\begin{array}{ll}1 & 3 \\ 0 & 5\end{array}\right]$ we get that there is only one normal matrix $N=\left[\begin{array}{ll}3 & 3 \\ 0 & 5\end{array}\right]$ such that $A=K N$ which is not hermitian.

(V) If $A$ is a normal $\{K, s+1\}$-potent matrix with $K^{2}=I, K^{*}=K$ satisfying $A^{s+3}=A$ then there is a hermitian matrix $H$ such that $A=K H$. Considering the same ring as in item (IV), it can be checked that the result is false by taking

$$
A=\left[\begin{array}{ll}
1 & 0 \\
0 & 3
\end{array}\right], \quad s=1, \quad K=I_{2} .
$$

(VI) If $A$ is a normal $K$-centro-symmetric matrix (that is, $A K=K A$ ) with $K^{2}=I, K^{*}=K$ then there is a normal matrix $N$ such that $A=K N$. This result is also valid in the ring (with involution) approach: If $a, k \in R$ satisfy $k^{2}=1, k^{*}=k, a$ is normal and $a k=k a$ then $k a$ is normal. In fact,

$$
(k a)^{*}(k a)=a^{*} k k a=k a^{*} a k=k a a^{*} k=(k a)(k a)^{*} .
$$


(VII) If $A$ is a $\{K, s+1\}$-potent matrix with $K^{2}=I, K^{*}=K$ satisfying $A^{*}=A^{(s+1)^{2}-1}$ then there is a normal matrix $N$ such that $A=K N$. This result is also valid in the ring (with involution) approach. In fact, by using the similar notation as in (VI) we get

$$
(k a)^{*}(k a)=a^{*} a=a^{(s+1)^{2}}=\left(a^{(s+1)^{2}}\right)^{s+1}=\left(a a^{*}\right)^{s+1}=a^{s+1}\left(a^{s+1}\right)^{*}
$$

and

$$
(k a)(k a)^{*}=a^{s+1} k\left(a^{s+1} k\right)^{*}=a^{s+1}\left(a^{s+1}\right)^{*} .
$$

We close this paper with the following questions:

- For the ring of complex matrices the following results are true: If $A$ is a normal $\{K, s+1\}$-potent matrix with $K^{2}=I, K^{*}=K$ then there is a normal matrix $N$ such that $A=K N$. Given a general ring $R$, does this statement remain valid?

- Can we drop the condition of the Dedekind finiteness of the ring in selected previous results?

\section{Acknowledgements}

The authors would like to thank the referees for their valuable comments and suggestions.

\section{References}

[1] R. Bru, N. Thome. Group inverse and group involutory matrices, Linear and Multilinear Algebra, 45 (2-3), 207-218, 1998.

[2] R.E. Hartwig, J. Luh, On finite regular rings, Pacific Journal Mathematics, 69, 73-95, 1977.

[3] R.E. Hartwig, P. Patrício, The link between regularity and strong-piregularity. Journal of Australian Mathematical Society, 89, 1, 17-22, 2010 .

[4] J.J. Koliha, D. Djordjević, D. Cvetković, Moore-Penrose inverse in rings with involution, Linear Algebra and its Applications, 426, 371-381, 2007. 
[5] L. Lebtahi, O. Romero, N. Thome, Characterizations of $\{K, s+1\}$ Potent Matrices and Applications. Linear Algebra and its Applications, 436, 293-306, 2012.

[6] L. Lebtahi, O. Romero, N. Thome, Relations between $\{K, s+1\}$-potent matrices and different classes of complex matrices. Linear Algebra and its Applications, DOI: 10.1016/j.laa.2011.10.042.

[7] A.R. Meenakshi, S. Krishnamoorthy, On k-EP matrices, Linear Algebra and its Applications, 269, 219-232, 1998.

[8] D. Mosić, D.S. Djordjević, J.J. Koliha, EP elements in rings, Linear Algebra and its Applications, 431, 5-7, 527-535, 2009. 